\title{
Clinical significance of electrocardiographic markers of myocardial damage prior to aortic valve replacement
}

\author{
Augustin Coisne a,b,c,d,*, Sandro Ninni b,c,d,e , François Pontana a,c,d,f , Samy Aghezzaf a , Florent Janvier ${ }^{\text {a }}$, \\ Anne-Laure Madika ${ }^{a}$, Bertrand Boutie ${ }^{a}$, Mohamad Koussa ${ }^{g}$, Antoine Bical ${ }^{g}$, André Vincentelli ${ }^{g}$, \\ Francis Juthier ${ }^{\mathrm{g}}$, Valentin Loobuyck ${ }^{\mathrm{g}}$, Arnaud Sudre ${ }^{\mathrm{e}}$, Stella Marchetta ${ }^{\mathrm{h}, \mathrm{i}}$, Christophe Martinez ${ }^{\mathrm{h}, \mathrm{i}}$, \\ Bart Staels ${ }^{\mathrm{b}, \mathrm{c}, \mathrm{d}, \mathrm{j}}$, Patrizio Lancellotti ${ }^{\mathrm{h}, \mathrm{i}}$, Thomas Modine ${ }^{\mathrm{g}}$, David Montaigne ${ }^{\mathrm{a}, \mathrm{b}, \mathrm{c}, \mathrm{d}}$ \\ a CHU Lille, Department of Clinical Physiology and Echocardiography, France \\ ${ }^{\mathrm{b}}$ Univ. Lille, U1011 - EGID, F-59000 Lille, France \\ c Inserm, U1011, F-59000 Lille, France \\ d Institut Pasteur de Lille, F-59000 Lille, France \\ e CHU Lille, Department of Cardiovascular Medicine, France \\ ${ }^{\mathrm{f}} \mathrm{CHU}$ Lille, Department of Cardiovascular Radiology, France \\ g CHU Lille, Department of Cardiovascular Surgery, France

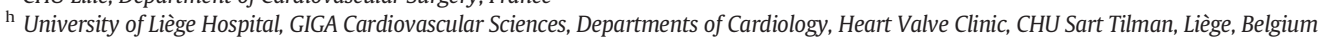

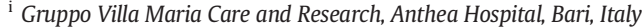 \\ ${ }^{j}$ CHU Lille, Department of Biology, France
} Stéphanie Mouton ${ }^{\mathrm{a}}$, Hélène Ridon ${ }^{\mathrm{a}}$, Staniel Ortmans ${ }^{\mathrm{a}}$, Claire Seunes ${ }^{\mathrm{a}}$, Marine Wautier ${ }^{\mathrm{a}}$, Amandine Coppin ${ }^{\mathrm{a}}$,

\section{A R T I C L E I N F O}

\section{Article history:}

Received 2 July 2019

Received in revised form 13 January 2020

Accepted 28 January 2020

Available online 29 January 2020

Keywords:

ECG strain

Aortic stenosis

Aortic valve replacement

Myocardial damage

\begin{abstract}
A B S T R A C T
Background: Pre-operative myocardial fibrosis and remodeling impact on outcomes after aortic valve replacement (AVR). We aimed at investigating the prognostic impact of preoperative electrocardiographic (ECG) markers of left ventricular (LV) myocardial damage, i.e. bundle branch block (BBB) and ECG strain pattern after (surgical or transcatheter) AVR for severe aortic stenosis (AS).

Methods: Between April 2008 and October 2017, we explored consecutive patients referred to our Heart Valve Clinic for first AVR for severe AS. Detailed pre-operative phenotyping and ECG analysis were performed. Patients were followed-up after AVR for major cardiac events (ME), i.e. cardiovascular death, cardiac hospitalization for acute heart failure and stroke.

Results: BBB and ECG strain were respectively observed in 13.5 and $21 \%$ of the 1122 patients included. These ECG markers identified a subgroup of older patients, with higher NYHA class and more advanced myocardial disease as detected by echocardiography, i.e. higher LV mass and lower LV ejection fraction, global longitudinal strain and integrated backscatter, than patients without ECG strain or BBB. ME occurred in $212(18.6 \%)$ patients during a mean follow-up of $4.4 \pm 1.5$ years with higher incidence in case of ECG strain or BBB (HR $1.56,95 \% \mathrm{CI}$ $1.13-2.14, p=0.006$; HR $1.47,95 \%$ CI 1.02-2.13, $p=0.04$ respectively). The prognostic value of ECG strain remained significant after adjustment for age, diabetes and pre-operative LVEF.

Conclusions: Pre-operative ECG markers of myocardial damage identify a subgroup of AS patients at high risk of post-AVR cardiovascular complications irrespective of other prognostic factors and should help the multiparametric staging of cardiac damage to guide AVR.
\end{abstract}

(C) 2020 Elsevier B.V. All rights reserved.

\section{Introduction}

Abbreviations: AS, Aortic Stenosis; AVR, Aortic Valve Replacement; ECG, Electrocardiogram; GLS, Global Longitudinal Strain; IBS, Integrated backscatter; LVH, A-Left Ventricular Hypertrophy; LVEF, Left Ventricular Ejection Fraction; NYHA, New - York Heart Association.

0- * Corresponding author at: Department of Clinical Physiology and r- Echocardiography, France - Heart Valve Clinic, Institut Coeur Poumon, CHU Lille, tic 559000 , France.

E-mail address: augustin.coisne@chru-lille.fr (A. Coisne). valve replacement (AVR) is the only treatment that proved efficacy to reduce morbi-mortality in symptomatic patients with severe aortic stenosis (AS) [1,2]. Nevertheless, one third of the patients remains symptomatic [3-5] or does not improve their functional capacities after surgery [6,7]. This lack of improvement is believed to reflect accumulating myocardial damage, secondary to the long-standing pressure overload. In AS, the chronic increased afterload is known to induce compensatory left ventricular hypertrophy (LVH), to maintain normal 
wall stress and cardiac function, and progressive myocardial fibrosis, both associated with adverse outcomes after AVR [8-10]. Recent findings regarding cardiac fibrosis and imaging have not been translated into daily practice since cardiac magnetic resonance imaging (MRI) is hardly accessible and speckle-tracking strain in transthoracic echocardiography (TTE) is not feasible in a substantial proportion of patients with AS due to poor echogenicity or atrial fibrillation. Unlike these imaging modalities, electrocardiogram (ECG) is a reliable and widespread tool to quickly assess the markers of a pathological myocardium. In line, a particular electrographic pattern called ECG strain has been described as a specific marker of mid-wall myocardial fibrosis on late gadolinium enhancement and T1 mapping cardiac MRI [11]. This pattern has been associated with an increased risk of cardiovascular mortality and morbidity in asymptomatic severe AS [11,12] and after isolated surgical AVR [13]. The fifth of patients with severe AS and presenting preoperative intraventricular conduction abnormalities, i.e. left or right bundle branch block (LBBB or RBBB), were excluded in studies exploring the ECG strain pattern. To date, there is no large prospective study exploring the long-term prognostic impact of ECG markers of myocardial damage (i.e. intraventricular conduction abnormalities and ECG strain) after surgical or transcatheter AVR for severe AS. We hypothesized patients with pre-operative ECG markers of ventricular myocardial damage to be at high risk of post-AVR cardiovascular complications irrespective of other prognostic factors.

\section{Methods}

\subsection{Study population and design}

From April 2008 to October 2017, we prospectively studied consecutive patients with severe AS referred to our Heart Valve Clinic (CHU Lille) for an AVR [14,15]. The operative technique (surgical or transcatheter) was based on current guidelines and left to the discretion of the Heart Team. Patients with another significant valvular disease, ventricular paced rhythm, a medical history of previous cardiac surgery or congenital heart disease were excluded. Informed consent was obtained from each patient and the study protocol conforms to the ethical guidelines of the 1975 Declaration of Helsinki.

\subsection{Transthoracic echocardiography}

A comprehensive pre-operative TTE including assessment of the aortic valve, was performed before AVR according to current guidelines [16] using state-of-the-art echocardiographic ultrasound systems (Vivid 7, Vivid E9 or Vivid E95, GE Healthcare, Little Chalfont, UK). From 2014, ultrasonic myocardial tissue characterization for the LV was implemented in routine: 2D speckle-tracking with systolic deformation analysis and integrated backscatter (IBS) were performed. IBS was obtained by placing region of interest in the mid-myocardium of anterior septum in parasternal long-axis view. IBS was then calibrated by subtracting pericardial IBS intensity as previously descried. [17]

\subsection{Electrocardiogram}

A standard pre-operative 12-lead ECG was recorded at $25 \mathrm{~mm} / \mathrm{s}$ and $1 \mathrm{mV} / \mathrm{cm}$ for all participants. ECG interpretation was performed and adjudicated by several observers (AC, SN, FP, FJ, SM, HR, SO, CS, MW, AC, ALM, BB \& DM) blinded to clinical and outcome data. LVH was defined by a Romhilt-Estes score $\geq$ ( 5 points) or a Sokolow-Lyon index $>3.5 \mathrm{mV}$ [18] and the ECG strain was defined as $\geq 0.1 \mathrm{mV}$ concave downsloping ST-segment depression with asymmetrical T-wave inversion in the lateral leads (I, aVL, V5, V6) as previously described. [19]

Baseline conduction abnormalities (left bundle branch block (LBBB), right bundle branch block (RBBB)) were also recorded and defined in accordance with the American Heart Association recommendations [20]. Our population was divided in 3 groups according to the following
ECG patterns: group $1=$ no ECG strain pattern, group $2=$ ECG strain pattern, group $3=$ baseline conduction abnormalities.

\subsection{Follow-up}

Patients were followed-up by direct patient interview and clinical examination, telephone calls with the physicians, patients or next of kin, or review of the autopsy records and death certificates. The following cardiac major events (ME) were recorded: cardiovascular deaths, cardiac hospitalizations for acute heart failure and stroke [21]. Allcause mortality was also recorded. Any event was adjudicated by two independent investigators blinded to the index clinical and echocardiographic data.

\subsection{Statistical analysis}

Based on previous literature, we hypothesized that a third of AS patients would display hallmark of cardiac damage, a rate of ME at 5 years of $25 \%$ in patients without ECG strain and a rate of $35 \%$ in patients with hallmark of myocardial damage/fibrosis [8-22]. This hypothesis implied that we had to analyse outcomes in at least 918 patients to obtain a statistical power of $90 \%$ and a probability of a type I error of 0.05 as previously described [23]. Continuous variables were tested for normality with Shapiro test, and were given as mean \pm SD. Categorical variables were given as percentages of individuals. Patients were separated in three groups according to pre-operative ECG patterns. One-way ANOVA analysis of variance was used for comparison of the three

A. Global longitudinal strain according to pre-operative ECG

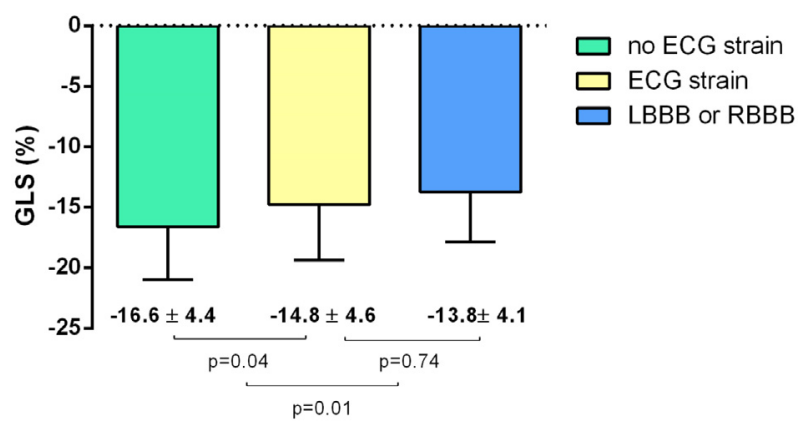

B. Integrated backscatter according to pre-operative ECG

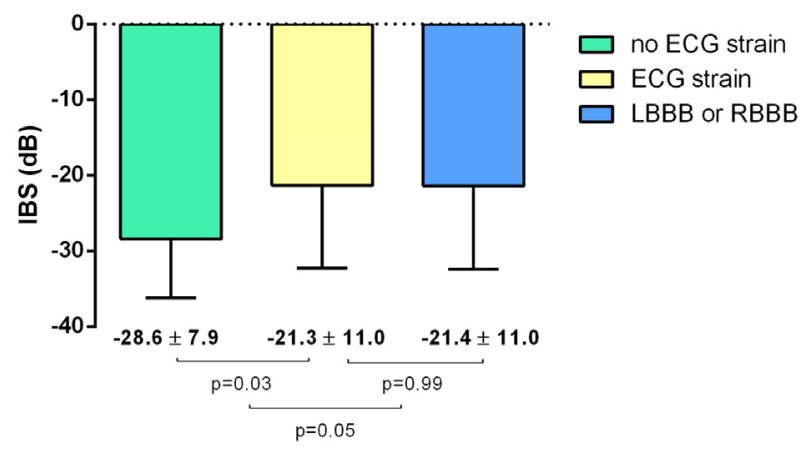

Fig. 1. Ultrasonic myocardial characterization. Comparison of A. Global longitudinal strain and B. Integrated backscatter according to pre-operative ECG in the patients included since 2014. p-Value by post-hoc t-test with Bonferroni correction. Mean \pm SD. A. Global longitudinal strain according to pre-operative ECG. B. Integrated backscatter according to pre-operative ECG. 
groups with Bonferroni post hoc t-test. Time-related clinical events were plotted with Kaplan-Meier curves according to ECG strain and conduction abnormalities definition and compared with log-rank tests. Cox proportional-hazards regression stepwise model was used to determine whether ECG strain and conduction abnormalities were associated with ME after adjusting for potential confounding variables. Variables with a value of $p<0.10$ on univariable analysis were incorporated into the multivariable model. A $p$ value $<0.05$ was considered statistically significant. Unless specified, statistics were performed using MedCalc v16.4 (Olstead, Belgium).

\section{Results}

3.1. Pre-operative ECG markers of ventricular myocardial damage in severe symptomatic AS

A total of 1122 consecutive patients referred for AVR were included. Mean age was $73 \pm 11$ years. The population was made of $56 \%$ of male, one fourth had diabetes and the large majority (91\%) was symptomatic (New York Heart Association (NYHA) class $\geq 2$ ). The mean LV ejection fraction (EF) was $59 \pm 10 \%$ and $78 \%$ underwent surgical aortic valve replacement (SAVR). ECG strain pattern was found in 236 patients (21\%) and conduction abnormalities in 152 patients (13.5\%). Characteristics of the population according to pre-operative ECG patterns are summarized in Table 1. A study flow chart is given in Supplemental Fig. 1.

Patients with ECG fibrosis/damage hallmarks were older, more frequently men, with more severe symptoms (NYHA stage), with more advanced myocardial disease as reflected by higher LV mass index (LVMi), larger left atrium area together with lower LVEF, global longitudinal strain global (GLS) (respectively $-14.8 \pm 4.6$ and $-13.8 \pm 4.1$ vs.
$-16.6 \pm 4.4 \%, p=0.003$ ) and IBS (respectively $-21.3 \pm 11.0$ and $-21.4 \pm 11.0$ vs. $-28.6 \pm 7.9 \mathrm{~dB}, p=0.02$ ) (Fig. $1 \&$ Supplemental Fig. 2). Accordingly, patients with ECG strain displayed a higher prevalence of ECG LVH assessed by Romhilt-Estes score (72 vs. 20\%, $p<0.0001$ ) or Sokolow-Lyon index (19 vs. $3 \%, p<0.0001$ ). Atrial fibrillation and concomitant coronary artery bypass graft surgery were respectively present in $10 \%$ and $20 \%$ of the population with a similar prevalence in the 3 groups.

\subsection{Prognostic impact of pre-operative ECG markers of ventricular myocar- dial damage}

The mean follow-up was $4.4 \pm 1.5$ years. ME occurred in 212 patients (18.9\%) with 113 cardiovascular deaths (10\%), 89 cardiac hospitalizations for acute heart failure (8.0\%) and 54 strokes (4.8\%). Allcause deaths occurred in 257 patients (23.0\%).

Both pre-operative ECG strain and conduction abnormalities were significantly associated with increase occurrence of ME in comparison with patients free from ECG strain, respectively (HR (95\% confidence interval, CI): 1.56 (1.13-2.14), $p=0.006$, and $1.47(1.02-2.13), p=0.04)$ (Fig. 2A). While conduction abnormalities were significantly associated with both cardiovascular death and all-cause mortality, $\mathrm{HR}(95 \% \mathrm{CI})$ respectively: 2.31 (1.49-3.6), $p=0.0002$ and 1.49 (1.08-2.07), $p=0.015$, ECG strain was not ( $\mathrm{HR}(95 \% \mathrm{CI})$ respectively: $1.27(0.79-2.04), p=0.29$ and 1.10 (0.85-1.58), $p=0.34$ ) (Fig. 2B, C and Supplemental Fig. 3).

On Cox univariable analysis, the occurrence of ME at 1500 days was significantly associated with old age, diabetes, hypertension, stroke, NYHA class, low pre-operative LVEF and mean aortic gradient, atrial fibrillation and the two ECG hallmarks of LV myocardial damage (Table 2). After multivariable adjustment using Cox regression analysis

Table 1

Patients' characteristics according to pre-operative ECG $(n=1122)$.

\begin{tabular}{|c|c|c|c|c|c|}
\hline & All population $(\mathrm{n}=1122)$ & No ECG strain $(n=734)$ & ECG strain $(n=236)$ & Conduction abnormalities $(n=152)$ & p-Value \\
\hline \multicolumn{6}{|l|}{ Pre-operative data } \\
\hline Age (years) & $72.9 \pm 10.7$ & $72.8 \pm 10.7$ & $71.1 \pm 11.2$ & $76.5 \pm 9.3^{*}, \#$ & $<0.001$ \\
\hline Gender male n (\%) & $628(56.0)$ & $384(51.5)$ & $161(68.2)$ & $83(54.6)$ & 0.0001 \\
\hline \multicolumn{6}{|l|}{ BMI $\left(\mathrm{kg} / \mathrm{m}^{2}\right)$} \\
\hline Smoker n (\%) & $234(20.9)$ & $144(19.6)$ & $65(27.5)$ & $25(16.4)$ & 0.009 \\
\hline Diabetes mellitus n (\%) & $306(27.3)$ & $203(27.7)$ & $68(28.8)$ & $35(23.0)$ & 0.42 \\
\hline Hypertension n (\%) & $729(65.0)$ & $481(65.5)$ & $151(64.0)$ & $97(63.8)$ & 0.86 \\
\hline Stroke n (\%) & $83(7.4)$ & $45(6.1)$ & $22(9.3)$ & $16(10.5)$ & 0.08 \\
\hline PAD n $(\%)$ & $108(9.6)$ & $71(9.7)$ & $23(9.8)$ & $14(9.2)$ & 0.85 \\
\hline CAD n $(\%)$ & $229(20.4)$ & $140(19.1)$ & $62(26.3)$ & $27(17.8)$ & 0.38 \\
\hline NYHA n (\%) & & & & & 0.0008 \\
\hline 1 & $97(8.6)$ & $72(9.8)$ & $17(7.2)$ & $8(5.3)$ & \\
\hline 2 & $631(56.2)$ & $419(57.1)$ & $130(55.1)$ & $82(53.9)$ & \\
\hline 3 & $317(28.3)$ & $211(28.7)$ & $59(25.0)$ & $47(30.9)$ & \\
\hline 4 & 77 (6.9) & $32(4.4)$ & $30(12.7)$ & $15(9.9)$ & \\
\hline \multicolumn{6}{|l|}{ TTE data } \\
\hline LVEF (\%) & $59.1 \pm 9.6$ & $60.3 \pm 8.4$ & $56.2 \pm 12.1^{*}$ & $58.8 \pm 9.9^{*}$ & $<\mathbf{0 . 0 0 1}$ \\
\hline Max aortic velocity (m/s) & $4.40 \pm 0.68$ & $4.4 \pm 0.6$ & $4.5 \pm 0.8$ & $4.3 \pm 0.6$ & 0.07 \\
\hline Mean gradient (mmHg) & $50.6 \pm 13.3$ & $50.7 \pm 12.3$ & $52.6 \pm 15.7^{*}$ & $50.1 \pm 13.5$ & 0.035 \\
\hline $\operatorname{LVMi}\left(\mathrm{g} / \mathrm{m}^{2}\right)$ & $117.9 \pm 33.0$ & $111.7 \pm 29.8$ & $130.8 \pm 36.4^{*}$ & $127.9 \pm 33.4^{*}$ & $<0.001$ \\
\hline LA surface $\left(\mathrm{cm}^{2}\right)$ & $25.8 \pm 7.9$ & $24.9 \pm 8.0$ & $27.1 \pm 8.2^{*}$ & $28.2 \pm 5.7^{*}$ & 0.004 \\
\hline \multicolumn{6}{|l|}{ ECG data } \\
\hline Atrial fibrillation n (\%) & $118(10.5)$ & $84(11.4)$ & $18(7.6)$ & $16(10.5)$ & 0.25 \\
\hline LVH Romhlit-Estes n (\%) & $319(28.4)$ & $149(20.3)$ & $170(72.0)$ & - & $<0.0001$ \\
\hline LVH Sokolow-Lyon n (\%) & $69(6.1)$ & $25(3.4)$ & $44(18.6)$ & - & $<0.0001$ \\
\hline \multicolumn{6}{|l|}{ Operative data } \\
\hline SAVR n (\%) & $848(78.3)$ & $549(74.8)$ & $163(69.1)$ & $106(69.7)$ & 0.0019 \\
\hline Concomitant CABG n (\%) & $219(19.5)$ & $148(20.2)$ & $47(19.9)$ & $24(15.8)$ & 0.51 \\
\hline
\end{tabular}

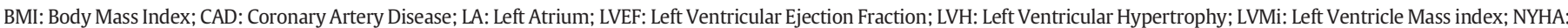
New York Heart Association; PAD: Peripheral Artery Disease; SAVR: Surgical Aortic Valve Replacement.

$p$-Value by one-way anova analysis of variance with Bonferroni post hoc paired $t$-test.

Bold values indicates statistically significance at $\mathrm{p}<0.05$.

* For $p<0.05$ by post-hoc t-test vs no ECG strain.

\# For $\mathrm{p}<0.05$ by post-hoc $t$-test vs ECG strain. 

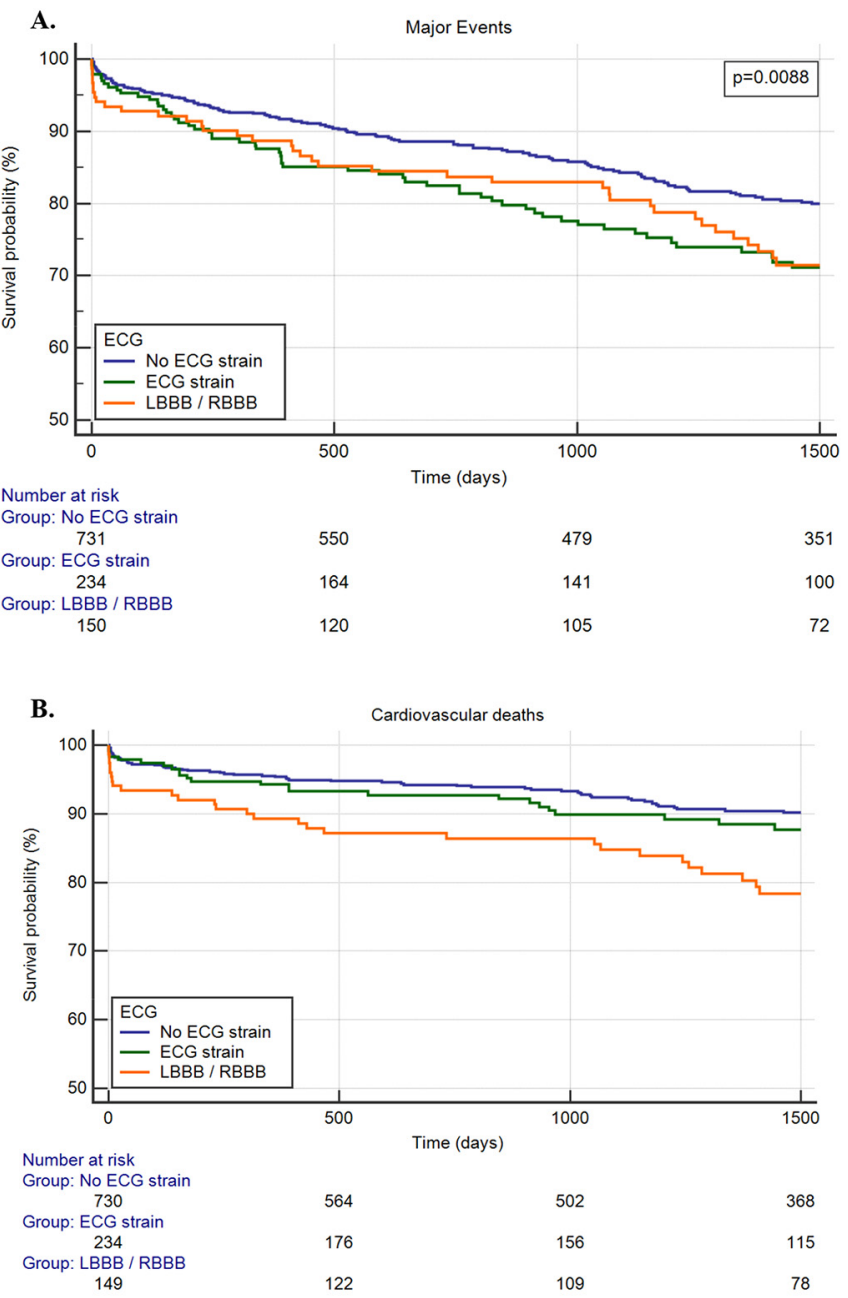

C.

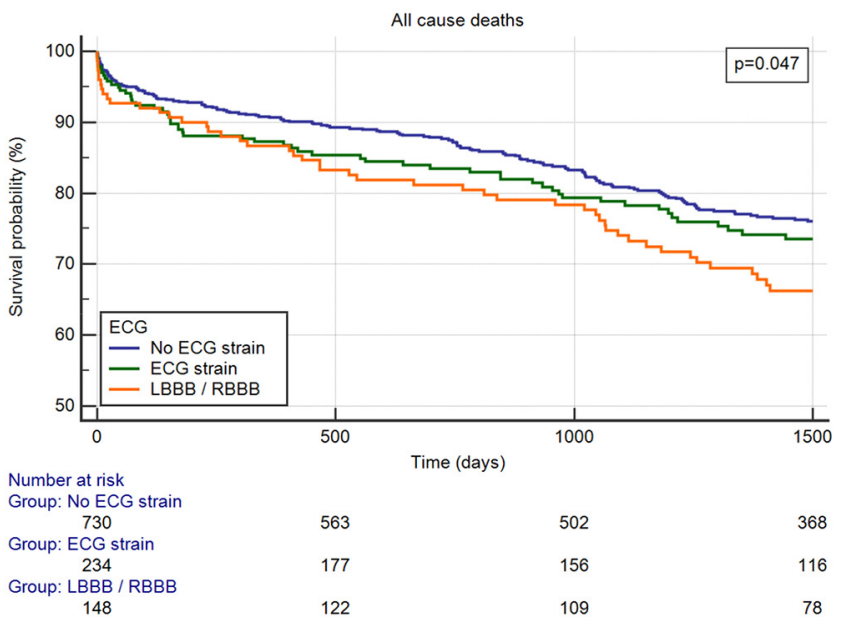

Fig. 2. Kaplan-Meier Survival Curves. Long-Term prognostic impact of pre-operative ECC markers of ventricular myocardial damage. Event-free survival from A) Major Events (cardiovascular death, stroke or acute heart failure), B) Cardiovascular Deaths and C) All Cause Deaths, according to pre-operative ECG. p-Value by log-rank test.

with stepwise selection of variables, old age, diabetes, high NYHA class, low LVEF and the presence of ECG strain were independently associated with long-term occurrence of $\mathrm{ME}$ ( $p=0.44$ and Chi-squared $=7.98$ by
Hosmer-Lemeshow test). ECG strain was predictive of ME independently of the type of AVR (HR: 1.40 (1.02-1.92); $p=0.03$ ). Intraventricular conduction abnormalities were not retained in the model.

\section{Discussion}

Exploring the clinical impact of pre-operative ECG markers of ventricular myocardial damage in a large cohort of patients with severe AS undergoing AVR, we demonstrated that ECG strain and conduction abnormalities were frequent, i.e. respectively in $21 \%$ and $13.5 \%$ of patients. This was consistent with previous studies reporting prevalence of 14 to $31 \%$ of this ECG features. [11-13]. Importantly, both ECG strain and conduction abnormalities were associated with long-term cardiac major events with conduction abnormalities also associated with allcause and cardiovascular deaths. The presence of pre-operative ECG strain was independently associated with long-term occurrence of ME together the commonly reported factors of poor prognosis after AVR, i.e. old age, diabetes, high NYHA class and low LVEF $[22,23]$.

To date, AVR is recommended in patients with severe AS and symptoms or with a LVEF $<50 \%[1,2]$. However, recent studies by Lancellotti et al. [22] and Tribouilloy et al. [23] demonstrated that patients with a LVEF respectively $<60 \%$ and $<55 \%$ are associated with an increased morbi-mortality even after AVR, suggesting that early (surgical or transcatheter) AVR should be considered to avoid irreversible myocardial damages. There is therefore a growing interest in studying the myocardial consequences of this valvular heart disease. We proposed here the simple ECG as an easy but efficient tool to improve multi-parametric risk stratification of patients prior to AVR, and thus refining the optimal timing of AVR by focusing on the myocardial repercussion of AS.

Although its physiopathology remains debated, previous studies showed that ECG strain is associated with an advanced hypertrophic response to AS with mid-wall fibrosis [12]. Accordingly, we showed that patients with ECG hallmarks of myocardial damage were older, more frequently men, with more severe symptoms, lower LVEF, higher LVMi and larger left atrium area. Moreover, in line with studies identifying GLS and IBS as tools to detect increased myocardial fibrosis [17,23], these 2 parameters exploring the LV were significantly depressed in AS patients with BBB or ECG strain pattern.

In addition of being a marker of a more advanced disease, ECG strain was independently associated with poor outcomes, corroborating recent findings by Magne et al. [13].

Based on our findings, multiparametric stratification of patients with severe AS prior to AVR should integrate hallmarks of ventricular myocardial damage. BBB or ECG strain pattern should encourage consideration for early intervention at the time of low peri-operative morbimortality associated with both surgical and trans-catheter AVR.

\subsection{Limitations}

Assessment of myocardial fibrosis was restricted to TTE parameters since cardiac magnetic resonance (MRI) was not used routinely in our hospital in this population. The relative prognostic insights of MRI hallmarks of ventricle damage on top of the widely available ECG and TTE should be tested in future studies. We sought to investigate preoperative ECG markers, and not postoperative results of AVR (surgical or transcatheter), in order to help the preoperative multiparametric staging of cardiac damage to guide AVR. Accordingly, we decided not to include peri-operative and post-operative characteristics in the survival model.

\subsection{Clinical perspectives}

ECG is a reliable and widespread tool to easily identify a subgroup of patients with extensive anatomical and functional cardiac damage secondary to AS that translate into higher incidence of cardiac events after 
Table 2

Univariable and multivariable Cox analyses to assess determinants of ME after AVR.

\begin{tabular}{|c|c|c|c|c|c|c|}
\hline & \multicolumn{3}{|c|}{ Univariable analysis } & \multicolumn{3}{|c|}{ Multivariable analysis } \\
\hline & p-Value & $\mathrm{HR}(95 \% \mathrm{CI})$ & $\beta \pm S E$ & p-Value & $\mathrm{HR}(95 \% \mathrm{CI})$ & $\beta \pm S E$ \\
\hline Age (years) & $<\mathbf{0 . 0 0 0 1}$ & & $0.04 \pm 0.008$ & 0.008 & & $0.02 \pm 0.008$ \\
\hline Gender n (\%) & 0.38 & $1.13(0.86-1.49)$ & & & & \\
\hline $\operatorname{BMI}\left(\mathrm{kg} / \mathrm{m}^{2}\right)$ & 0.43 & & $0.01 \pm 0.01$ & & & \\
\hline Smoker n (\%) & 0.73 & $1.06(0.76-1.48)$ & & & & \\
\hline Diabetes mellitus n (\%) & 0.001 & $1.61(1.21-2.13)$ & & 0.0006 & $1.65(1.24-2.20)$ & \\
\hline Hypertension n (\%) & 0.04 & $1.37(1.02-1.83)$ & & - & - & - \\
\hline Stroke n (\%) & 0.05 & $1.57(1.00-2.47)$ & & - & - & - \\
\hline NYHA n (\%) & $<0.0001$ & $1.77(1.48-2.13)$ & & 0.037 & $1.23(1.01-1.50)$ & \\
\hline $\operatorname{LVEF}(\%)$ & $<\mathbf{0 . 0 0 0 1}$ & & $-0.03 \pm 0.007$ & - & & - \\
\hline Mean aortic gradient $(\mathrm{mmHg})$ & 0.01 & & $-0.01 \pm 0.006$ & - & - & \\
\hline $\operatorname{LVMi}\left(\mathrm{g} / \mathrm{m}^{2}\right)$ & 0.12 & & $0.004 \pm 0.002$ & & & \\
\hline Atrial fibrillation n (\%) & 0.0001 & $1.87(1.45-2.95)$ & & 0.02 & $1.54(1.07-2.23)$ & \\
\hline LVH Romhilt-Estes n (\%) & 0.22 & $1.21(0.89-1.65)$ & & & & \\
\hline LVH Solow-Lyon n (\%) & 0.23 & $0.72(0.53-1.16)$ & & & & \\
\hline \multicolumn{7}{|l|}{ ECG signs of fibrosis n (\%) } \\
\hline ECG strain & 0.006 & $1.56(1.13-2.14)$ & & 0.03 & $1.40(1.02-1.92)$ & \\
\hline Conduction abnormalities & 0.04 & $1.47(1.01-2.13)$ & & - & - & - \\
\hline SAVR n (\%) & $<0.0001$ & $0,29(0,22-0,38)$ & & $<0.0001$ & $0,44(0,31-0,62)$ & \\
\hline
\end{tabular}

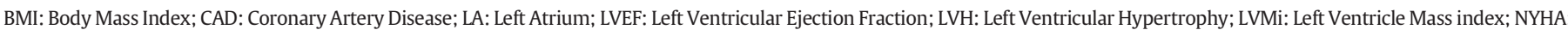
New York Heart Association; PAD: Peripheral Artery Disease; SAVR: Surgical Aortic Valve Replacement. Bold values indicates statistically significance at $\mathrm{p}<0.05$.

AVR. It should help the multiparametric staging of cardiac damage to guide AVR.

\section{Conclusion}

BBB or ECG strain is observed in a third of AS patients referred for AVR. These ECG markers of myocardial damage identify a subgroup of patients with extensive anatomical and functional cardiac damage secondary to AS that translate into higher incidence of cardiac events after AVR.

Supplementary data to this article can be found online at https://doi. org/10.1016/j.ijcard.2020.01.073.

\section{Declaration of competing interest}

No disclosures to declare.

\section{References}

[1] H. Baumgartner, V. Falk, J.J. Bax, M. De Bonis, C. Hamm, P.J. Holm, et al., 2017 ESC/ EACTS guidelines for the management of valvular heart disease, Eur Heart J. 38 (36) (2017 Sep 21) 2739-2791.

[2] R.A. Nishimura, C.M. Otto, R.O. Bonow, B.A. Carabello, J.P. Erwin, L.A. Fleisher, et al., 2017 AHA/ACC focused update of the 2014 AHA/ACC guideline for the management of patients with valvular heart disease: a report of the American College of Cardiology/American Heart Association Task Force on Clinical Practice Guidelines, Circulation 135 (25) (2017 Jun 20) e1159-e1195.

[3] H.J. Lamb, H.P. Beyerbacht, A. de Roos, A. van der Laarse, H.W. Vliegen, F. Leujes, et al., Left ventricular remodeling early after aortic valve replacement: differential effects on diastolic function in aortic valve stenosis and aortic regurgitation, J. Am. Coll. Cardiol. 40 (12) (2002 Dec 18) 2182-2188.

[4] F. Weidemann, S. Herrmann, S. Störk, M. Niemann, S. Frantz, V. Lange, et al., Impact of myocardial fibrosis in patients with symptomatic severe aortic stenosis, Circulation. 120 (7) (2009 Aug 18) 577-584.

[5] M. Gilard, H. Eltchaninoff, B. Iung, P. Donzeau-Gouge, K. Chevreul, J. Fajadet, et al., Registry of transcatheter aortic-valve implantation in high-risk patients, N. Engl. J. Med. 366 (18) (2012 May 3) 1705-1715.

[6] T.A. Treibel, R. Kozor, R. Schofield, G. Benedetti, M. Fontana, A.N. Bhuva, et al., Reverse myocardial remodeling following valve replacement in patients with aortic stenosis, J. Am. Coll. Cardiol. 71 (8) (2018 Feb 27) 860-871.

[7] O. Abdul-Jawad Altisent, R. Puri, A. Regueiro, C. Chamandi, T. Rodriguez-Gabella, M. Del Trigo, et al., Predictors and association with clinical outcomes of the changes in exercise capacity after transcatheter aortic valve replacement, Circulation. 136 (7) (2017 Aug 15) 632-643.

[8] D.A. Orsinelli, G.P. Aurigemma, S. Battista, S. Krendel, W.H. Gaasch, Left ventricular hypertrophy and mortality after aortic valve replacement for aortic stenosis. A high risk subgroup identified by preoperative relative wall thickness, J. Am. Coll. Cardiol. 22 (6) (1993 Nov 15) 1679-1683.

[9] C.W.L. Chin, D. Messika-Zeitoun, A.S.V. Shah, G. Lefevre, S. Bailleul, E.N.W. Yeung, et al., A clinical risk score of myocardial fibrosis predicts adverse outcomes in aortic stenosis, Eur. Heart J. 37 (8) (2016 Feb 21) 713-723.

[10] S. Herrmann, B. Fries, T. Salinger, D. Liu, K. Hu, D. Gensler, et al., Myocardial fibrosis predicts 10-year survival in patients undergoing aortic valve replacement, Circ Cardiovasc Imaging. 11 (8) (2018 Aug), e007131.

[11] A.S.V. Shah, C.W.L. Chin, V. Vassiliou, S.J. Cowell, M. Doris, T.C. Kwok, et al., Left ventricular hypertrophy with strain and aortic stenosis, Circulation. 130 (18) (2014 Oct 28) 1607-1616.

[12] A.M. Greve, K. Boman, C. Gohlke-Baerwolf, Y.A. Kesäniemi, C. Nienaber, S. Ray, et al., Clinical implications of electrocardiographic left ventricular strain and hypertrophy in asymptomatic patients with aortic stenosis: the simvastatin and ezetimibe in aortic stenosis study, Circulation. 125 (2) (2012 Jan 17) 346-353.

[13] B. Guinot, J. Magne, A. Le Guyader, E. Bégot, A. Bourgeois, A. Piccardo, et al., Usefulness of electrocardiographic strain to predict survival after surgical aortic valve replacement for aortic stenosis, Am. J. Cardiol. 120 (8) (2017 Oct 15) 1359-1365.

[14] A. Coisne, T. Modine, F. Outteryck, S. Mouton, R. Pilato, H. Ridon, et al., Clinical significance of right ventricular longitudinal function parameters after aortic valve replacement, JACC Cardiovasc. Imaging 11 (4) (2018 Apr) 651-652.

[15] A. Coisne, S. Ninni, J.-L. Edmé, T. Modine, S. Mouton, R. Pilato, et al., Obesity paradox in the clinical significance of effective prosthetic orifice area after aortic valve replacement, JACC Cardiovasc. Imaging 12 (1) (2019 Jan) 208-210.

[16] R.M. Lang, L.P. Badano, V. Mor-Avi, J. Afilalo, A. Armstrong, L. Ernande, et al. Recommendations for cardiac chamber quantification by echocardiography in adults: an update from the American Society of Echocardiography and the European Association of Cardiovascular Imaging, J Am Soc Echocardiogr. 28 (1) (2015 Jan) 1-39(e14).

[17] V. Di Bello, D. Giorgi, P. Viacava, T. Enrica, C. Nardi, C. Palagi, et al., Severe aortic stenosis and myocardial function: diagnostic and prognostic usefulness of ultrasonic integrated backscatter analysis, Circulation. 110 (7) (2004 Aug 17) 849-855.

[18] D.W. Romhilt, E.H. Estes, A point-score system for the ECG diagnosis of left ventricular hypertrophy, Am. Heart J. 75 (6) (1968 Jun) 752-758.

[19] E.W. Hancock, B.J. Deal, D.M. Mirvis, P. Okin, P. Kligfield, L.S. Gettes, et al., AHA/ACCF HRS recommendations for the standardization and interpretation of the electrocardiogram: part V: electrocardiogram changes associated with cardiac chamber hypertrophy: a scientific statement from the American Heart Association Electrocardiography and Arrhythmias Committee, Council on Clinical Cardiology; the American College of Cardiology Foundation; and the Heart Rhythm Society. Endorsed by the International Society for Computerized Electrocardiology, J. Am. Coll. Cardiol. 53 (11) (2009 Mar 17) 992-1002.

[20] B. Surawicz, R. Childers, B.J. Deal, L.S. Gettes, J.J. Bailey, A. Gorgels, et al., AHA/ACCF/ HRS recommendations for the standardization and interpretation of the electrocardiogram: part III: intraventricular conduction disturbances: a scientific statement from the American Heart Association Electrocardiography and Arrhythmias Committee, Council on Clinical Cardiology; the American College of Cardiology Foundation; and the Heart Rhythm Society. Endorsed by the International Society for Computerized Electrocardiology, J. Am. Coll. Cardiol. 53 (11) (2009 Mar 17) 976-981.

[21] K.A. Hicks, J.E. Tcheng, B. Bozkurt, B.R. Chaitman, D.E. Cutlip, A. Farb, et al., 2014 ACC AHA key data elements and definitions for cardiovascular endpoint events in clinical 
trials: a report of the American College of Cardiology/American Heart Association Task Force on Clinical Data Standards (writing committee to develop cardiovascular endpoints data standards), J Am Coll Cardiol. 66 (4) (2015 Jul 28) 403-469.

22] P. Lancellotti, J. Magne, R. Dulgheru, M.-A. Clavel, E. Donal, M.A. Vannan, et al., Outcomes of patients with asymptomatic aortic stenosis followed up in heart valve clinics, JAMA Cardiol. 3 (11) (2018 Nov 1) 1060-1068.
[23] Y. Bohbot, A. Pasquet, D. Rusinaru, J. Delabre, O. Delpierre, A. Altes, et al., Asymptomatic severe aortic stenosis with preserved ejection fraction: early surgery versus conservative management, J. Am. Coll. Cardiol. 72 (23 Pt A) (2018 Dec 11) 2938-2939. 